\title{
AN APPROACH OF NETWORK CODE DIFFERENTIAL GPS POSITIONING FOR MEDIUM AND LONG DISTANCES
}

\author{
M. Bakuła \\ University of Warmia and Mazury in Olsztyn, \\ Chair of Satellite Geodesy and Navigation, Poland. \\ E-mail:mbakula@uni.olsztyn.pl
}

\begin{abstract}
This contribution presents an approach of network code DGPS post-processing positioning with the use of at least three simultaneous reference stations. The traditional DGPS is based on only one reference station. Using only one reference station, a rover receiver does not have redundant observations. The accuracy of DGPS positioning is very difficult to estimate and, in some DGPS receivers, is not valid or the DGPS positions are determined without estimated accuracy. In practical experiments worldwide, it has been shown that code DGPS accuracy varies from approximately 1 to 2 meters depending on the number of satellites and their configuration. This paper presents new functional and stochastic models of network DGPS positioning for medium and long distances, allowing centimeter accuracy for some epochs with valid accuracy estimation based on variance covariance (VC) matrix. Valid accuracy estimation is an extremely important issue from a practical point of view for allowing large enhance of DGPS applications. Practical calculations and analysis of the approach have been presented with the use of GPS data from the ASG-PL (Polish Active Geodetic Network) network.
\end{abstract}

\section{INTRODUCTION}

Differential code GPS technique is used to improve the autonomous positioning of a rover station by applying corrections provided by a GPS base/reference station. The reference station calculates pseudorange corrections (PRC) that are applied to the remote receiver in near real time (real-time DGPS) or after GPS measurements (post-processing DGPS). In order to provide a differential positioning service for a large area, several reference stations have to be used. The approach of multiple reference stations network is known as wide-area DGPS (WADGPS), for example WAAS (Wide Area Augmentation System) or EGNOS (European Geostationary Navigation Overlay Service).

Differential correction techniques are used to enhance the quality of location data gathered using GPS receivers. There are many permanent GPS base stations currently operating throughout the world that provide the data necessary for differentially correcting GPS. Depending on the technology preferred by the base station owner, this data can be downloaded from the Internet or GSM/GPRS technology. Base station data is rather consistent and very reliable because base stations usually operate 24 hours a day, and it is very convenient for many GIS and mapping applications. These reference stations are also used for network RTK (Real Time Kinematic) positioning elaborated in the last decade by several research groups (e.g., Wanninger, 1995; Wübbena et al., 1996; Wanninger, 1997; Raquet, 1997; Lachapelle et al., 2000; Landau et al., 2002; Euler et al., 2001; Chen, 2004; 
Kashani et al., 2004). The models presented in these studies concern mostly carrier-phase positioning where high accuracy $(\mathrm{cm}$ or $\mathrm{mm})$ is expected. To achieve this, the ambiguity resolution process should be first performed. However, in the case of DGPS we do not need to calculate ambiguities due to the fact that only pseudorange measurements are applied.

The traditional DGPS positioning approach was studied in many papers worldwide where practical tests show that accuracy of code differential positioning is within 1-2 $\mathrm{m}$ and sometimes even more. Due to the degradation of standard DGPS positioning by distancedependent errors, this paper presents the approach of linear interpolation and the smoothing process of determined positions. Additionally, a weight matrix is proposed for valid and reliable accuracy estimation of determined positions. The calculations presented in the paper are based on data from Ashtech $\mu$ Z-CGRS, a state-of-the-art, low noise, 12-channel GPS receiver.

\section{PSEUDORANGE CORRECTIONS FOR A VIRTUAL REFERENCE STATION}

If there are three reference stations (e.g. REF1, REF2, REF3) in the vicinity of an unknown control point, virtual reference pseudorange corrections can be calculated using linear interpolation. This process reduces distance-dependent errors and some non-systematic errors. Three pseudorange corrections generate a correlation plane for every satellite, thus the pseudorange (PRC) corrections for every satellite can be presented according to the following equations:

$$
\begin{aligned}
& a(t) x_{R E F 1}+b(t) y_{R E F 1}+c(t)=P R C_{R E F 1}(t), \\
& a(t) x_{R E F 2}+b(t) y_{R E F 2}+c(t)=P R C_{R E F 2}(t), \\
& a(t) x_{R E F 3}+b(t) y_{R E F 3}+c(t)=P R C_{R E F 3}(t) .
\end{aligned}
$$

For three reference stations, the factors $a(t), b(t), c(t)$ are calculated for each epoch according to the matrix equation:

$$
\left[\begin{array}{l}
a(t) \\
b(t) \\
c(t)
\end{array}\right]=\left[\begin{array}{lll}
x_{R E F 1} & y_{R E F 1} & 1 \\
x_{R E F 2} & y_{R E F 2} & 1 \\
x_{R E F 3} & y_{R E F 3} & 1
\end{array}\right]^{-1}\left[\begin{array}{l}
P R C_{R E F 1}(t) \\
P R C_{R E F 2}(t) \\
P R C_{R E F 3}(t)
\end{array}\right]
$$

where $x_{R E F}, y_{R E F}$ are planar coordinates.

For $n$ reference stations, using the least-squares solution, the matrices $A$ (design matrix) and $L$ (observed minus calculated matrix) are as follows:

$$
\begin{aligned}
\mathrm{A} & =\left[\begin{array}{ccc}
x_{R E F 1} & y_{R E F 2} & 1 \\
\vdots & \vdots & \vdots \\
x_{R E F n} & y_{R E F n} & 1
\end{array}\right] \\
\mathrm{L}^{\mathrm{T}} & =\left[\begin{array}{lll}
P R C_{R E F 1}(t) & \cdots & P R C_{R E F n}(t)
\end{array}\right]
\end{aligned}
$$

The pseudorange correction for the satellite $k$ at the reference station REF1 is given by (e.g. Hofmann - Wellenhof et al. 1997)

$$
P R C_{R E F 1}^{k}(t)=\rho_{R E F 1}^{k}(t)-P_{R E F 1}^{k}(t)
$$

In previous work (Bakuła, 2005), the values of pseudorange corrections were calculated based on three reference stations and the lineal interpolation process. Next, a smoothing procedure 
was used to model pseudorange corrections. From that flowchart, one step is removed, concerning smoothing of VRS corrections and a new flowchart is created as in Fig. 1. It had to be done in order to introduce valid weights of observations analogously, as it was presented for two reference stations (Bakuła, 2004).

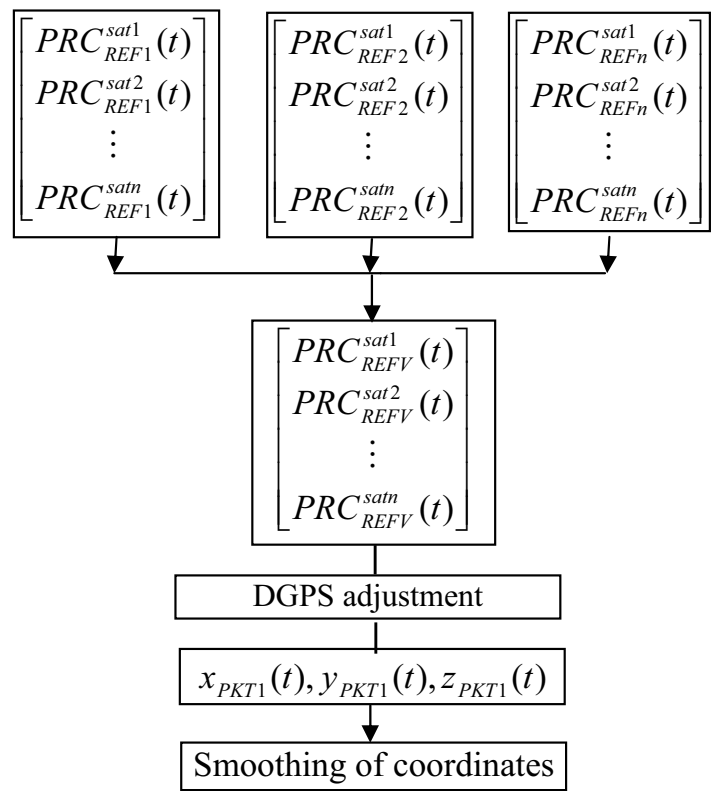

Fig. 1. Flowchart of the network code differential GPS positioning

Finally, after the DGPS adjustment, the adjusted coordinates were smoothed by the supsmooth function of Mathcad 11 software. The supsmooth function uses a symmetric $k$ nearest neighbor linear least-squares fitting procedure to make a series of line segments through the data, and chooses different bandwidths for different portions of the data.

\section{EQUATIONS OF DGPS POSITIONING AND ACCURACY ESTIMATION}

Let the vector of observation be denoted by $\mathrm{L}$, the vector of unknowns by $\mathrm{X}$ and the design matrix by A. Then, employing the least-squares method, the estimator of the unknown vector $\hat{\mathrm{X}}$ equals:

$$
\hat{\mathrm{X}}=\left(\mathrm{A}^{\mathrm{T}} \mathrm{PA}\right)^{-1} \mathrm{~A}^{\mathrm{T}} \mathrm{PL}
$$

It is known that the values of $\hat{X}$ depend on functional and stochastic models. The stochastic model represented by a weight matrix plays an essential role in accuracy estimation during the adjustment solution. Many different approaches can be found in literature, corresponding to various special applications of GPS methods used in practice. In the present DGPS postprocessing positioning using the least-squares solution, the matrices of Eq. 6 are as follows:

$$
\mathrm{A}=\left[\begin{array}{ccc}
a_{X i}^{\text {sat } 1} & a_{Y i}^{\text {sat } 1} & a_{Z i}^{\text {sat } 1} \\
\vdots & \vdots & \vdots \\
a_{X i}^{\text {satn }} & a_{Y i}^{\text {satn }} & a_{Z i}^{\text {satn }}
\end{array}\right],
$$




$$
\begin{aligned}
\mathrm{L} & =\left[\begin{array}{c}
l_{i}^{\text {sat } 1} \\
\vdots \\
l_{i}^{\text {satn }}
\end{array}\right], \\
\mathrm{P} & =\operatorname{diag}\left[p_{1} \cdots p_{n}\right],
\end{aligned}
$$

where: $a_{Z i}^{\text {satn }}=-\frac{\mathrm{x}^{\text {satn }}(t)-\mathrm{x}_{\mathrm{i} 0}}{\rho_{\mathrm{i} 0}^{\text {satn }}(\mathrm{t})}$,

$$
\begin{aligned}
& a_{Y i}^{\text {satn }}=-\frac{\mathrm{x}^{\text {satn }}(t)-\mathrm{x}_{\mathrm{i} 0}}{\rho_{\mathrm{i} 0}^{\text {satn }}(\mathrm{t})}, \\
& a_{Z i}^{\text {satn }}=-\frac{\mathrm{x}^{\text {satn }}(t)-\mathrm{x}_{\mathrm{i} 0}}{\rho_{\mathrm{i} 0}^{\text {satn }}(\mathrm{t})},
\end{aligned}
$$

$\mathrm{x}_{\mathrm{i} 0}, \mathrm{y}_{\mathrm{i} 0}, \mathrm{z}_{\mathrm{i} 0}$ - the approximate coordinates for the unknown station,

$\mathrm{x}^{\mathrm{k}}(\mathrm{t}), \mathrm{y}^{\mathrm{k}}(\mathrm{t}), \mathrm{z}^{\mathrm{k}}(\mathrm{t})$ the coordinates of the satellite $k, \rho_{i 0}^{k}(t)$ - geometric distance between the station and the satellite.

At the beginning of the presented DGPS positioning, a unity weight matrix was tested based on the assumption that the accuracy of pseudorange measurements obtained from particular satellites was the same.

Next, the following weights were used (for every satellite) as a function of VRS pseudorange corrections of reference stations $P R C_{R E F V}^{n}(t)$ and the rover station $P R C_{P K T 1}^{n}(t)$ :

$$
p_{n}=\frac{1}{\left[P R C_{R E F V}^{n}(t)-P R C_{P K T 1}^{n}(t)\right]^{2}}
$$

The weights of Eq. 10 reflect the linear behaviour of pseudorange corrections in network code DGPS positioning. The proposed matrices $\mathrm{L}$ and $\mathrm{P}$, in the iterative least-squares adjustment method can reduce time-spatial errors, pseudorange noise and, especially, the weight matrix can eliminate gross errors taking place at the reference stations and/or the rover station.

When iterative process of LS solution is finished, the statistics for the adjusted estimated unknown coordinates should be computed. The first item to be computed is the variance factor (Leick 1995)

$$
\sigma_{0}=\sqrt{\frac{\mathrm{V}^{\mathrm{T}} \mathrm{PV}}{n-m}}
$$

where: $\mathrm{V}=\mathrm{AX}-\mathrm{L}, n-m$ is the total redundancy of the system, $n$ represents number of unknowns but $m$ is the number of observations.

The second, the standard deviations for the estimated station coordinates should be computed. They are obtained from variance-covariance matrix of the adjusted parameters

$$
\Sigma_{\mathrm{X}}=\sigma_{0}^{2}\left(\mathrm{~A}^{\mathrm{T}} \mathrm{PA}\right)^{-1}
$$

\section{NUMERICAL EXAMPLES}

The GPS data used in this chapter were collected at seven known permanent reference stations of the ASG-PL (e.g. Polish Active Geodetic Network) network (Fig. 3): KLOB in 
Kłobuck, KATO in Katowice, WODZ in Wodzisław Śląski, TARG in Tarnowskie Góry, ZYWI in Żywiec, LELO in Lelów and CBKA in Warsaw, Poland (Kryński et al, 2003). The reference stations were equipped with Ashtech $\mu$ Z-CGRS (Continuous Geodetic Reference Station) receivers and ASH701945C_M SNOW antennas, except for the stations CBKA where another type of the antenna is used, i.e. ASH701945E M SNOW. The GPS observations were performed on 17 April 2005, from 13:00 to 13.20 UTC; the sampling interval was 5 seconds; the values of PDOP during the measurements were between two and four. Six satellites were used in the calculations.

The presented elaborations are divided into two cases:

1) for distances between reference stations up to about $100 \mathrm{~km}$; reference stations: KLOB, KATO, WODZ and the rover station TARG,

2) for distances between reference stations up to about $300 \mathrm{~km}$; reference stations: KLOB, CBKA, ZYWI and the rover station LELO.

In both cases, the calculations were performed as in the traditional DGPS positioning where one reference station is used; the results are depicted in Figs: $3 a, 3 b$ and $3 c$-for shorter distances and in Figs: 5a, 5b and 5c -for longer distances. Additionally, calculations were performed with the use of three reference stations, with the results presented in Figs $3 \mathrm{~d}, 3 \mathrm{e}, 3 \mathrm{f}$ and $5 \mathrm{~d}, 5 \mathrm{e}, 5 \mathrm{f}$-for both cases, respectively. In all approaches, real errors were calculated $(\Delta \mathrm{n}$, $\Delta \mathrm{e}, \Delta \mathrm{z})$ as the differences between the fixed coordinates of the rover stations (TARG, LELO) and coordinates obtained in successive epochs of differential positioning.

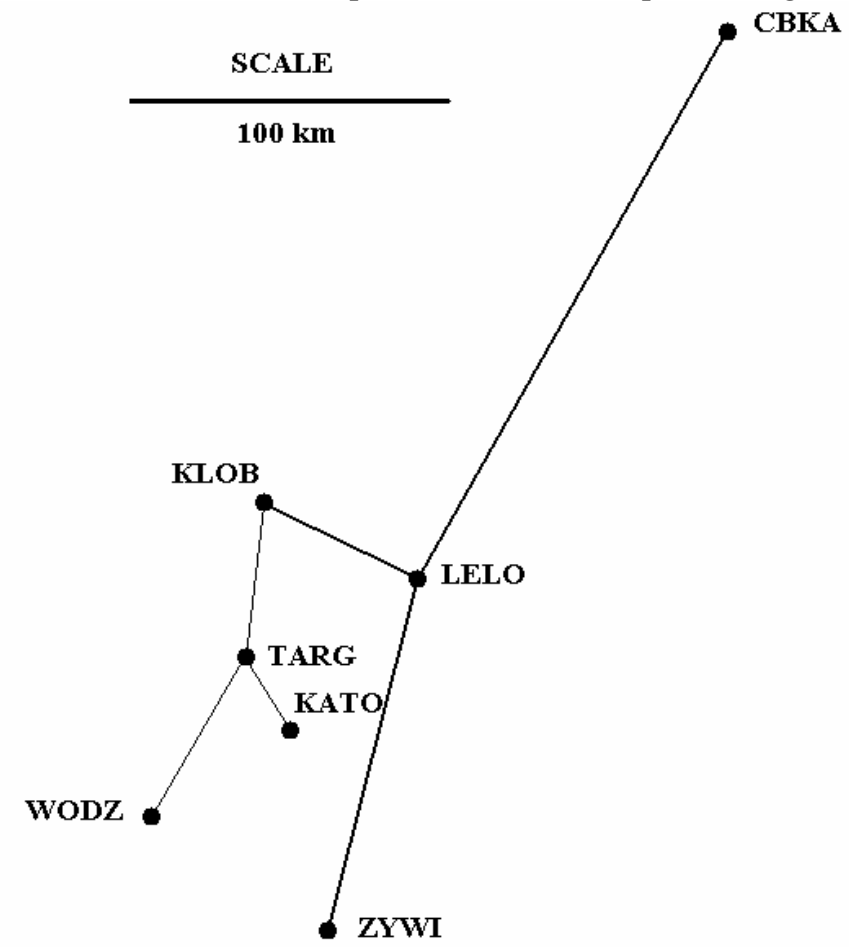

Fig. 2. Deployment of permanent reference stations of the ASG-PL network used in calculations 


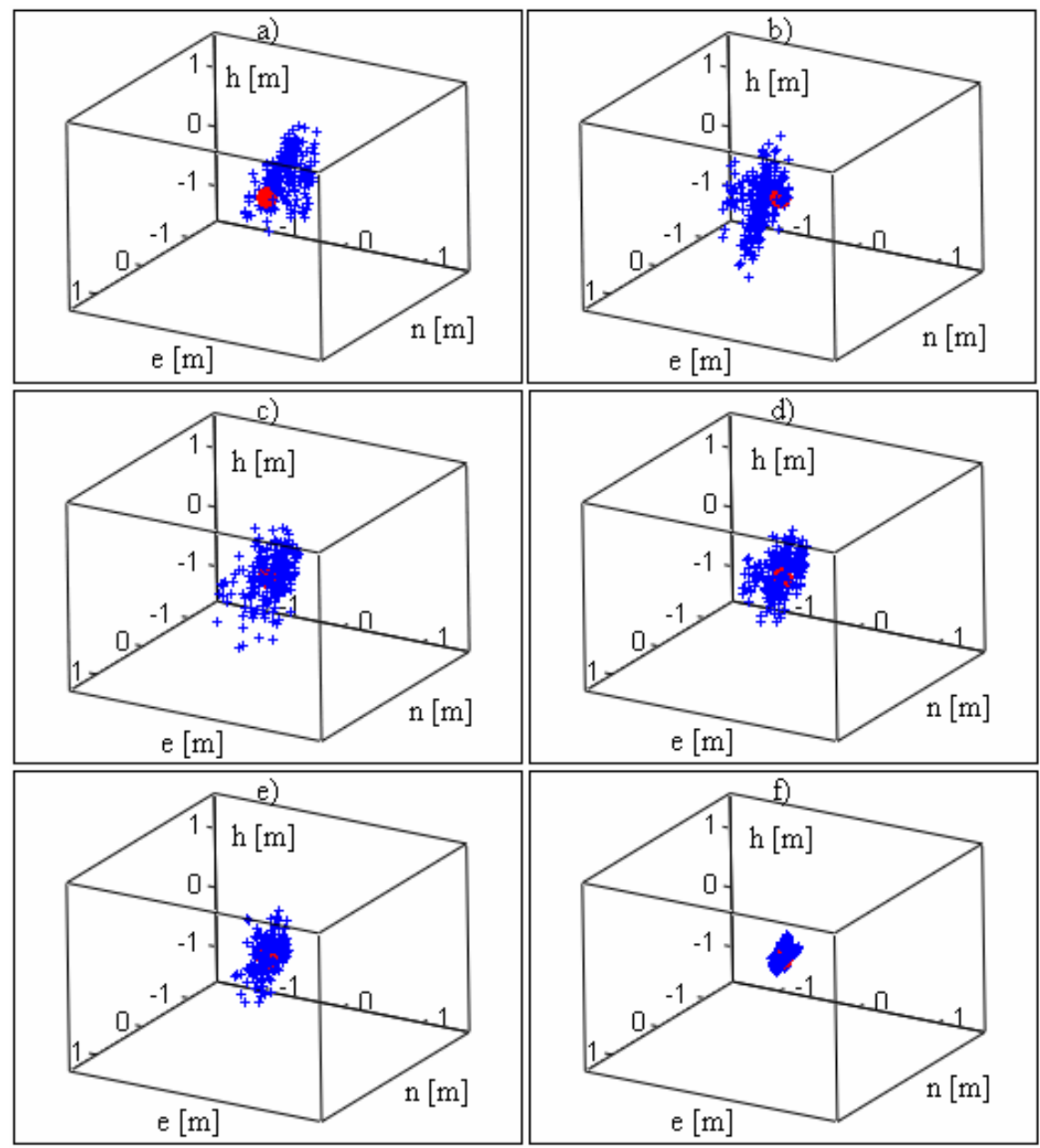

Fig. 3. Real errors of traditional DGPS positioning for different medium distances a) WODZ-

TARG, (58 km), b) KLOB-TARG, (51 km), c) KATO-TARG, (25 km); network DGPS solutions: d) with using linear interpolation, e) with linear interpolation and the weight matrix, f) with the use of smoothing procedures of adjusted coordinates

Some statistics of the traditional DGPS positioning are presented in the paper (Bakuła, 2005), where mean values with standard deviations were calculated. However, here only the values of accuracy estimated from the VC matrix can be focused on. The results are depicted in Fig 4. It can be seen that the variance factor is below unity in the traditional DGPS positioning (Figs $4 \mathrm{a}, 4 \mathrm{~b}, 4 \mathrm{c}$ ). It follows that treating pseudorange corrections as if they have the same accuracy is not quite true. In the case when the weight matrix was different from unity and represented by Eg. 10, one can see that the variance factor stayed around the unity (Fig. 4c). For a comparison of both approaches: traditional and network DGPS positioning, the socalled RMS difference $(\Delta r m s)$ term was introduced, calculated by the following equation

$$
\Delta r m s=\sqrt{\Delta n^{2}+\Delta e^{2}+\Delta h^{2}}-\sqrt{m_{n}^{2}+m_{e}^{2}+m_{h}^{2}}
$$

If the $\Delta r m s$ is around zero, it is better. It shows that the accuracy from the VC matrix is reliable. It can be seen that the accuracy from the VC matrix compared with real errors is much better in the network DGPS than in traditional DGPS positioning. It should be said that 
this consistency was in the range of a few centimeters for the variance factor in the range of from 0.9 to 1.1 .
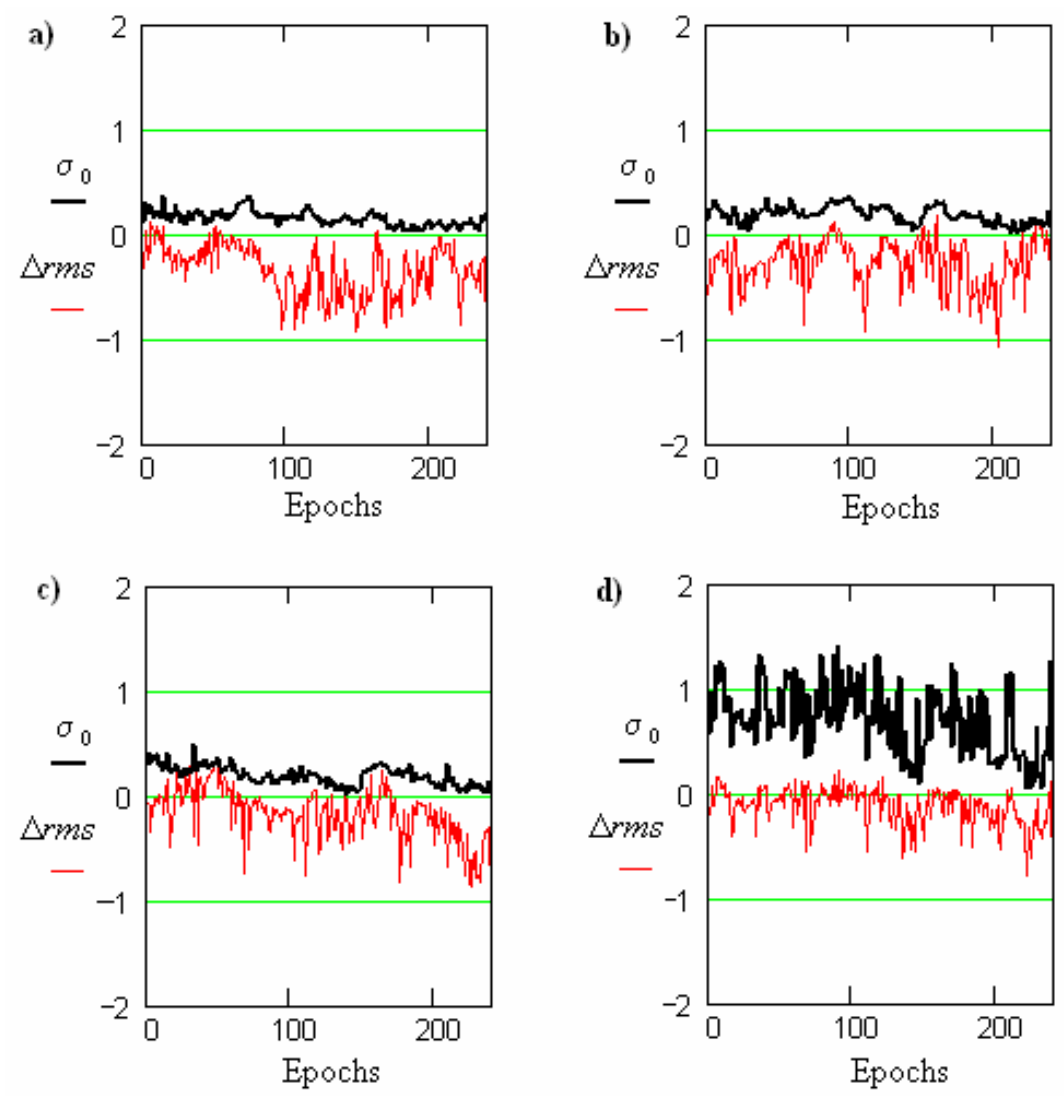

Fig. 4. Values of 3D RMS difference ( $\Delta r m s)$ and values of the variance factor obtained in different approaches of DGPS positioning: a) WODZ-TARG, (58 km), b) KLOB-TARG, (51 $\mathrm{km})$, c) KATO-TARG, (25 km); network DGPS solution: d) with linear interpolation and the weight matrix

In the second case (for longer distances) of numerical calculations, the influence of the network DGPS positioning against traditional DGPS is more visible. For longer distances in the traditional DGPS, positions are evidently apart from the true position e.g.:

- if the ZYWI station was used as the reference station, differences between the mean values and fixed coordinates were such as $\Delta \mathrm{n}=-0.45 \mathrm{~m}, \Delta \mathrm{e}=0.47 \mathrm{~m}$ and $\Delta \mathrm{h}=0.55 \mathrm{~m}$ (Fig. 5a);

- for KLOB-TARG: $\Delta \mathrm{n}=-0.26 \mathrm{~m}, \Delta \mathrm{e}=-0.20 \mathrm{~m}$ and $\Delta \mathrm{h}=0.07 \mathrm{~m}$ (Fig. 5b);

- if the CBKA-TARG: $\Delta \mathrm{n}=0.70 \mathrm{~m}, \Delta \mathrm{e}=-0.23 \mathrm{~m}$ and $\Delta \mathrm{h}=-0.35 \mathrm{~m}$ (Fig. 5c).

However, in the network DGPS, mean values of calculated coordinates agreed with true positions within centimeters. More interesting, however, is validation of the accuracy from the VC matrix. As shown in Figure 6, only when Eq. 10 determined the weight matrix were the values from the $\mathrm{VC}$ matrix strictly connected with the variance factor. For the variance factor from 0.9 to 1.1 , there was a very high convergence $(\Delta r m s)$ of estimated accuracy with real errors (Fig. 6c). 

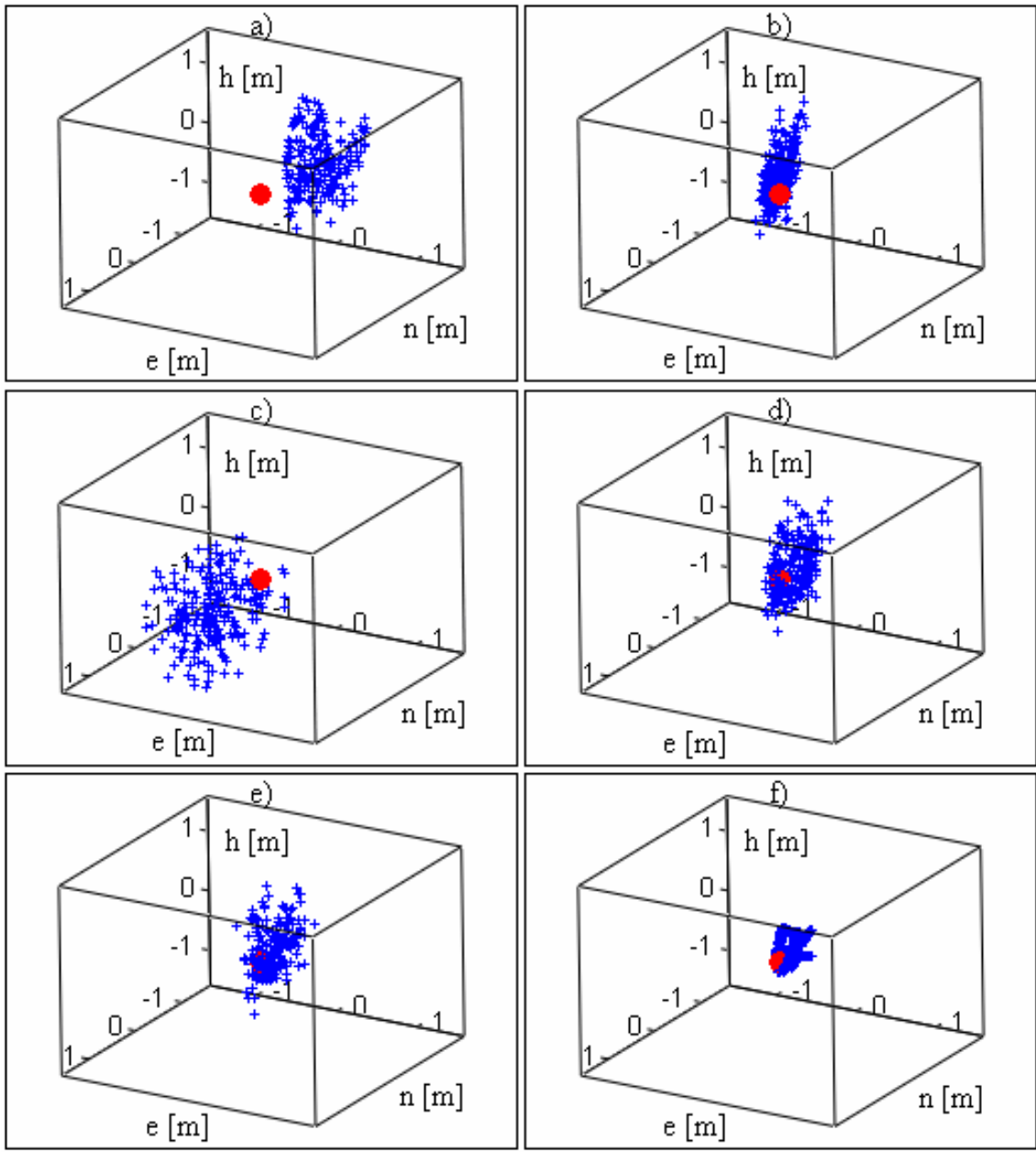

Fig. 5. Real errors of traditional DGPS positioning for different long distances: a) ZYWILELO, (115 km), b) KLOB-LELO, (55 km), c) CBKA-LELO, (198 km); network DGPS solutions: d) with using linear interpolation, e) with linear interpolation and the weight matrix, f) with the use of smoothing procedures of adjusted coordinates 

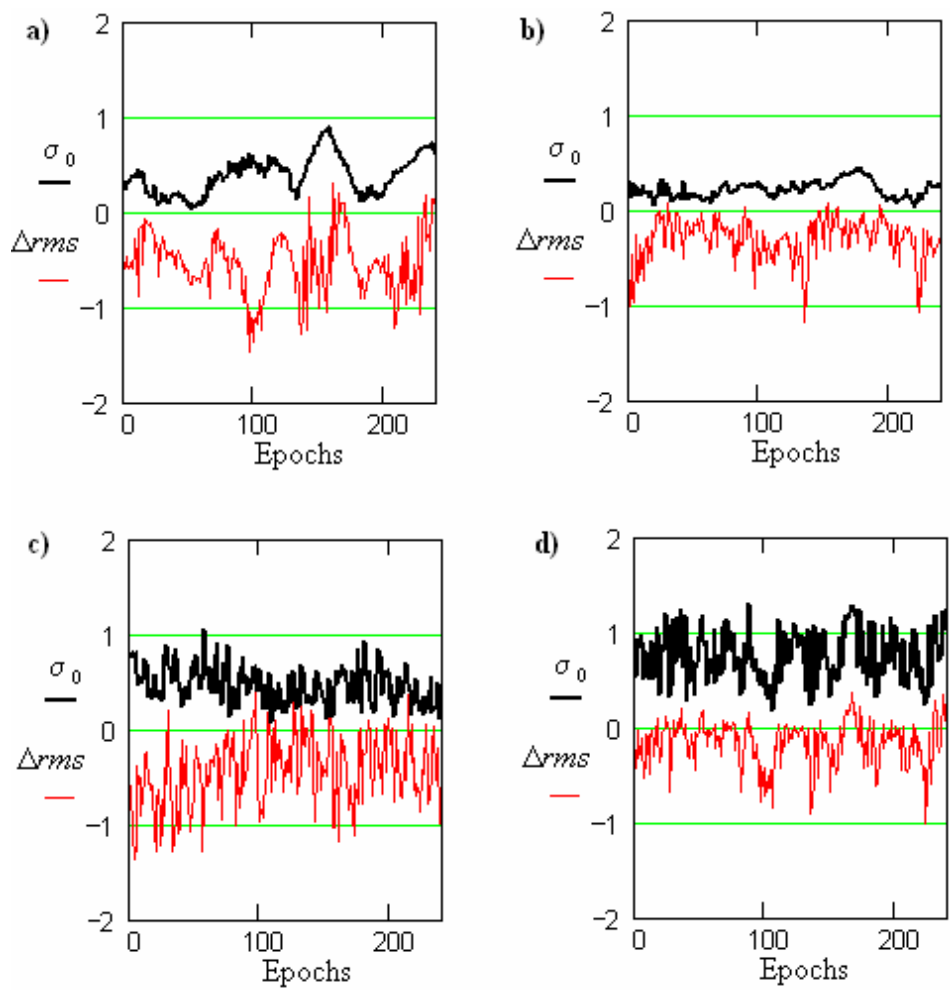

Fig. 6. Values of 3D RMS difference ( $\triangle r m s)$ and values of variance factor obtained in different approaches of DGPS positioning: a) ZYWI-LELO, (115 km), b) KLOB-LELO, (55 $\mathrm{km})$, c) CBKA-LELO, (198 km); network DGPS solution: d) with linear interpolation and the weight matrix

In both cases, for medium and long distances, the proposed functional and stochastic models substantially improved accuracy and allowed valid accuracy estimation based on the variance covariance matrix. Valid accuracy estimation is a key issue from a practical point of few and can allow more extensive use of code network DGPS solutions in practice.

\section{SUMMARY AND CONCLUSIONS}

In this paper, the linear interpolation approach was applied to generate pseudorange corrections of differential positioning with the use of the C/A code. Test data from the ASGPL network were used to evaluate the performance of this method. Each reference station was equipped with a state-of-the-art, low noise, 12-channel GPS receiver. The data obtained in the study show that the use of linear interpolation of pseudorange corrections could reduce distance-dependent errors in DGPS positioning. When the traditional method of DGPS positioning was used, the accuracies were different for every reference station. For the shortest distances between the reference and rover stations, e.g. KATO - TARG, the real accuracy was in the range of $1 \mathrm{~m}$, but for the longest distance (CBKA - LELO), the real accuracy was in the range of $2 \mathrm{~m}$. However, the application of the proposed method improved the accuracy of DGPS positioning and achieved accuracy in the range of 0.1-0.2 $\mathrm{m}$ for shorter distances and $0.2-0.5 \mathrm{~m}$ for the longer in every epoch. The main advantage of the proposed method is improved accuracy estimation based on the $\mathrm{VC}$ matrix. According to the $\mathrm{VC}$ matrix, epochs can be obtained where the accuracy is valid, e.g. the variance factor is close to unity. Tables 1 and 2 present coincidences of real accuracy (dn, de, dh) with accuracy 
estimation from the VC matrix $\left(\mathrm{m}_{\mathrm{n}}, \mathrm{m}_{\mathrm{e}}, \mathrm{m}_{\mathrm{h}}\right)$; in both cases, there were epochs were the variance factor was in the range of 0.9-1.1 and there are quite good coincidences of real and estimated accuracy (within centimeters). It should be noted, however, that there are epochs where the estimated centimeter accuracy agreed quite well with real errors, e.g. for epochs: $15,49,51,53,64,81,132,145,152,153,155,173,182,188$ in the first example of network DGPS positioning, and for epochs 61, 72, 115, 122, 123, 128, 174, 190, 200, 206 in the second experiment (for long distances) in network DGPS positioning.

Table 1. Values of variance factor $\left(\sigma_{0}\right)$, real errors $(\Delta \mathrm{n}, \Delta \mathrm{e}, \Delta \mathrm{h})$ and standard deviations $\left(\mathrm{m}_{\mathrm{n}}\right.$, $\mathrm{m}_{\mathrm{e}}, \mathrm{m}_{\mathrm{h}}$ obtained from VC matrix for medium distances of the network DGPS solution

\begin{tabular}{|c|c|c|c|c|c|c|c|}
\hline $\begin{array}{c}\text { No of } \\
\text { epoch }\end{array}$ & $\sigma_{0}$ & $\begin{array}{c}\Delta \mathrm{n} \\
{[\mathrm{m}]}\end{array}$ & $\begin{array}{c}\Delta \mathrm{e} \\
{[\mathrm{m}]}\end{array}$ & $\begin{array}{c}\Delta \mathrm{h} \\
{[\mathrm{m}]}\end{array}$ & $\mathrm{m}_{\mathrm{n}}[\mathrm{m}]$ & $\begin{array}{c}\mathrm{m}_{\mathrm{e}} \\
{[\mathrm{m}]}\end{array}$ & $\begin{array}{c}\mathrm{m}_{\mathrm{h}} \\
{[\mathrm{m}]}\end{array}$ \\
\hline 3 & 0.96 & 0.11 & -0.12 & -0.21 & 0.19 & 0.20 & 0.20 \\
\hline 6 & 1.05 & 0.01 & -0.04 & -0.13 & 0.16 & 0.19 & 0.19 \\
\hline 7 & 1.05 & 0.01 & -0.05 & -0.13 & 0.15 & 0.18 & 0.18 \\
\hline 11 & 1.03 & 0.02 & -0.03 & -0.10 & 0.10 & 0.12 & 0.12 \\
\hline 15 & 0.92 & -0.04 & 0.01 & -0.05 & 0.04 & 0.05 & 0.05 \\
\hline 40 & 1.03 & 0.07 & -0.07 & 0.07 & 0.09 & 0.11 & 0.12 \\
\hline 49 & 1.01 & 0.03 & 0.04 & 0.01 & 0.02 & 0.03 & 0.03 \\
\hline 51 & 0.99 & 0.04 & 0.05 & 0.04 & 0.03 & 0.05 & 0.04 \\
\hline 53 & 1.00 & -0.08 & 0.02 & 0.07 & 0.09 & 0.08 & 0.10 \\
\hline 55 & 1.09 & -0.10 & 0.01 & 0.06 & 0.12 & 0.09 & 0.13 \\
\hline 64 & 1.02 & -0.03 & 0.00 & 0.00 & 0.03 & 0.01 & 0.02 \\
\hline 65 & 1.09 & -0.06 & 0.00 & -0.02 & 0.15 & 0.04 & 0.05 \\
\hline 66 & 0.98 & -0.08 & -0.03 & -0.04 & 0.15 & 0.05 & 0.06 \\
\hline 69 & 0.94 & -0.03 & -0.02 & -0.06 & 0.16 & 0.04 & 0.06 \\
\hline 75 & 0.98 & 0.11 & 0.09 & 0.01 & 0.06 & 0.04 & 0.06 \\
\hline 81 & 1.09 & -0.09 & -0.04 & 0.02 & 0.07 & 0.02 & 0.04 \\
\hline 95 & 0.92 & -0.17 & 0.01 & -0.04 & 0.07 & 0.04 & 0.12 \\
\hline 97 & 1.01 & -0.30 & 0.07 & 0.20 & 0.35 & 0.15 & 0.20 \\
\hline 99 & 0.93 & -0.17 & -0.02 & 0.00 & 0.10 & 0.02 & 0.07 \\
\hline 100 & 0.93 & -0.08 & 0.02 & 0.11 & 0.15 & 0.04 & 0.08 \\
\hline 101 & 1.06 & -0.10 & 0.06 & 0.15 & 0.24 & 0.10 & 0.16 \\
\hline 102 & 0.99 & -0.09 & 0.03 & -0.15 & 0.16 & 0.15 & 0.14 \\
\hline 106 & 1.07 & -0.10 & -0.02 & 0.02 & 0.11 & 0.01 & 0.04 \\
\hline 125 & 0.91 & -0.15 & -0.05 & 0.06 & 0.11 & 0.01 & 0.04 \\
\hline 132 & 0.97 & -0.08 & -0.02 & 0.00 & 0.05 & 0.03 & 0.05 \\
\hline 145 & 0.91 & -0.06 & -0.01 & 0.06 & 0.06 & 0.02 & 0.04 \\
\hline 152 & 0.98 & 0.00 & 0.07 & 0.00 & 0.04 & 0.07 & 0.07 \\
\hline 153 & 1.04 & -0.04 & 0.01 & -0.03 & 0.01 & 0.02 & 0.05 \\
\hline 155 & 1.04 & -0.09 & 0.08 & -0.07 & 0.01 & 0.08 & 0.07 \\
\hline 156 & 0.96 & 0.01 & 0.06 & -0.13 & 0.08 & 0.06 & 0.14 \\
\hline 158 & 0.97 & 0.05 & 0.06 & -0.15 & 0.11 & 0.07 & 0.16 \\
\hline 159 & 0.99 & 0.04 & 0.07 & -0.15 & 0.11 & 0.07 & 0.16 \\
\hline 160 & 0.98 & -0.05 & 0.09 & -0.16 & 0.06 & 0.08 & 0.15 \\
\hline 161 & 0.97 & -0.01 & 0.07 & -0.15 & 0.08 & 0.07 & 0.17 \\
\hline 173 & 0.93 & 0.02 & -0.04 & -0.07 & 0.06 & 0.07 & 0.07 \\
\hline 182 & 0.93 & 0.09 & 0.04 & -0.06 & 0.07 & 0.04 & 0.03 \\
\hline 188 & 0.93 & 0.12 & 0.04 & -0.05 & 0.08 & 0.02 & 0.03 \\
\hline 189 & 1.00 & 0.15 & 0.03 & -0.07 & 0.11 & 0.04 & 0.04 \\
\hline 192 & 0.92 & 0.19 & 0.04 & -0.09 & 0.11 & 0.03 & 0.03 \\
\hline
\end{tabular}


Table 2. Values of variance factor $\left(\sigma_{0}\right)$, real errors $(\Delta \mathrm{n}, \Delta \mathrm{e}, \Delta \mathrm{h})$ and standard deviations $\left(\mathrm{m}_{\mathrm{n}}\right.$, $\mathrm{m}_{\mathrm{e}}, \mathrm{m}_{\mathrm{h})}$ obtained from VC matrix for long distances of the network DGPS solution

\begin{tabular}{|r|r|r|r|r|r|r|r|}
\hline $\begin{array}{r}\text { No of } \\
\text { epoch }\end{array}$ & $\sigma_{0}$ & $\begin{array}{c}\Delta \mathrm{n} \\
{[\mathrm{m}]}\end{array}$ & $\begin{array}{c}\Delta \mathrm{e} \\
{[\mathrm{m}]}\end{array}$ & $\begin{array}{c}\Delta \mathrm{h} \\
{[\mathrm{m}]}\end{array}$ & $\begin{array}{c}\mathrm{m}_{\mathrm{n}} \\
{[\mathrm{m}]}\end{array}$ & $\begin{array}{c}\mathrm{m}_{\mathrm{e}} \\
{[\mathrm{m}]}\end{array}$ & $\begin{array}{c}\mathrm{m}_{\mathrm{h}} \\
{[\mathrm{m}]}\end{array}$ \\
\hline 2 & 0.93 & 0.23 & 0.28 & 0.07 & 0.13 & 0.20 & 0.23 \\
\hline 5 & 0.97 & 0.26 & 0.49 & 0.19 & 0.32 & 0.22 & 0.29 \\
\hline 6 & 0.91 & 0.13 & 0.47 & 0.19 & 0.17 & 0.21 & 0.21 \\
\hline 17 & 1.05 & 0.01 & 0.10 & 0.04 & 0.12 & 0.07 & 0.15 \\
\hline 18 & 1.00 & 0.16 & 0.05 & -0.02 & 0.09 & 0.05 & 0.06 \\
\hline 26 & 0.96 & -0.15 & 0.02 & -0.10 & 0.08 & 0.10 & 0.12 \\
\hline 50 & 0.95 & -0.11 & 0.08 & -0.18 & 0.10 & 0.18 & 0.18 \\
\hline 53 & 0.90 & -0.02 & 0.04 & -0.10 & 0.06 & 0.02 & 0.06 \\
\hline 55 & 0.92 & -0.14 & -0.01 & -0.11 & 0.06 & 0.05 & 0.13 \\
\hline 61 & 1.03 & -0.03 & 0.02 & -0.04 & 0.07 & 0.02 & 0.05 \\
\hline 67 & 1.05 & 0.13 & 0.01 & 0.13 & 0.14 & 0.07 & 0.19 \\
\hline 72 & 1.03 & 0.09 & -0.07 & 0.08 & 0.01 & 0.09 & 0.08 \\
\hline 76 & 1.00 & 0.13 & 0.01 & 0.12 & 0.14 & 0.05 & 0.14 \\
\hline 115 & 1.00 & 0.02 & 0.03 & -0.01 & 0.02 & 0.02 & 0.02 \\
\hline 116 & 0.94 & -0.09 & 0.15 & 0.18 & 0.17 & 0.16 & 0.17 \\
\hline 121 & 0.93 & -0.30 & 0.07 & -0.23 & 0.12 & 0.15 & 0.19 \\
\hline 122 & 1.09 & -0.10 & -0.11 & 0.02 & 0.09 & 0.08 & 0.10 \\
\hline 123 & 1.08 & -0.06 & -0.10 & -0.05 & 0.08 & 0.07 & 0.09 \\
\hline 124 & 1.01 & -0.19 & -0.09 & 0.10 & 0.16 & 0.06 & 0.06 \\
\hline 127 & 0.95 & -0.25 & 0.05 & 0.16 & 0.22 & 0.15 & 0.14 \\
\hline 128 & 0.96 & -0.04 & 0.00 & 0.00 & 0.02 & 0.01 & 0.01 \\
\hline 129 & 0.97 & -0.12 & 0.02 & -0.11 & 0.11 & 0.10 & 0.09 \\
\hline 133 & 1.03 & -0.10 & 0.14 & 0.22 & 0.14 & 0.16 & 0.17 \\
\hline 138 & 1.07 & -0.23 & -0.18 & 0.05 & 0.19 & 0.12 & 0.19 \\
\hline 145 & 1.02 & -0.36 & -0.19 & 0.04 & 0.25 & 0.12 & 0.14 \\
\hline 161 & 0.93 & 0.15 & 0.20 & -0.19 & 0.18 & 0.20 & 0.25 \\
\hline 174 & 1.02 & 0.03 & 0.01 & -0.04 & 0.07 & 0.02 & 0.02 \\
\hline 190 & 1.07 & -0.07 & 0.06 & -0.03 & 0.08 & 0.05 & 0.08 \\
\hline 200 & 1.08 & -0.07 & -0.01 & 0.00 & 0.05 & 0.04 & 0.05 \\
\hline 201 & 0.92 & -0.31 & 0.05 & 0.13 & 0.22 & 0.09 & 0.12 \\
\hline 206 & 0.92 & 0.01 & -0.07 & 0.04 & 0.04 & 0.05 & 0.06 \\
\hline 214 & 1.02 & -0.27 & -0.04 & 0.15 & 0.24 & 0.10 & 0.21 \\
\hline 215 & 1.00 & -0.28 & 0.00 & 0.12 & 0.22 & 0.09 & 0.17 \\
\hline 226 & 1.08 & -0.35 & -0.23 & 0.01 & 0.22 & 0.18 & 0.21 \\
\hline 229 & 1.07 & -0.24 & -0.19 & -0.02 & 0.19 & 0.15 & 0.27 \\
\hline 238 & 1.03 & -0.26 & 0.10 & -0.20 & 0.26 & 0.25 & 0.20 \\
\hline 240 & 1.00 & -0.29 & 0.08 & -0.17 & 0.16 & 0.25 & 0.20 \\
\hline & & & & & & & \\
\hline
\end{tabular}

It should also be noted that for other epochs where the variance factor was in the range of 0.91.1 , the estimated accuracy from VC matrix also agreed with real errors quite well although the estimated errors are larger. It shows that this approach is reliable in terms of accuracy estimation in the network DGPS positioning. Such reliable estimated accuracy in DGPS positioning makes this technique useful not only for navigation, but also for many mapping applications of the Geographic Information Systems. Finally, it should be underlined that the 
presented calculations concerning network DGPS positioning were performed for every epoch independently. This approach seems to be very helpful for determining ambiguity searching domains for long baselines in carrier phase network positioning.

\section{REFERENCES}

Bakuła M. (2004): An Approach of DGPS Positioning Based on Two Reference Stations, Artificial Satellites, Journal of Planetary Geodesy, Vol. 39, No. 4.

Bakuła M. (2005): Static Code DGPS Positioning Based on Three Reference Stations, Geodesy and Cartography, Vol. 54, No 2, pp. 81-92.

Chen R., Li X. (2004): Virtual Differential GPS Based on SBAS Signal, GPS Solutions, Vol. 8, No. 4, pp. 238-244.

Euler H.J., Keenan C.R., Zebhauser B.E., Wübbena G. (2001): Study of Simplified Approach in Utilizing Information from Permanent Reference Station Arrays, Paper presented at ION GPS 2001, Salt Lake City, Utach.

Fotopoulos G., Cannon M.E. (2001): An Overview of Multi-Reference Station Methods for Cm-Level Positioning, GPS Solutions, Vol. 4,No. 3, pp. 1-10.

Hofmann - Wellenhof B., Lichtenegger H., Collins J. (1997): Global Positioning System: Theory and Practice, 4th ed. Springer, Berlin Heidelberg New York.

Kashani I., Grejner-Brzezińska D., Wielgosz P. (2004): Towards Instantaneous NetworkBased RTK GPS Over $100 \mathrm{~km}$ Distance, Proceedings of the ION $60^{\text {th }}$ Annual Meeting, June 7-9, Dayton, Ohio.

Kryński J., Rogowski J.B., Zieliński J.B. (2003): National Report of Poland to EUREF 2003, EUREF Symposium, Toledo, Spain, 4-7 June.

Lachapelle G., Alves P., Fortes L.P., Cannon M.E, Townsend B. (2000): DGPS RTK Positioning Using a Reference Network, Proceedings of GPS2000 (Session C3, Salt Lake City, 19-22 September), The Institute of Navigation, Alexandria, VA.

Landau H., Vollath U., Chen X. (2002): Virtual Reference Stations Systems, Journal of Global Positioning Systems, Vol. 1, No 2, pp 137-143.

Leick A. (1995): GPS Satellite Surveying, 2nd ed. John Wiley \& Sons, New York.

Raquet, J. (1997): A New Approach to GPS Carrier Phase Ambiguity Resolution Using a Reference Receiver Network, Proceedings of National Technical Meeting, Santa Monica, January 14-16), The Institute of Navigation, Alexandria, VA, 357-366.

Wanninger L. (1995): Improved Ambiguity Resolution by Regional Differential Modelling of the Ionosphere, Proc. of ION GPS 95, Palm Springs.

Wanninger L. (1997): Real-Time Differential GPS-Error Modelling in Regional Reference Station Networks in: Brunner, F.K. (Hg.): Advances in Positioning and Reference Frames, Proceedings of the IAG Scientific Assembly, Rio de Janeiro.

Wübbena G., Bagge A., Seeber G., Böder V., Hankemeier P. (1996): Reducing Distance Dependent Errors for Real-Time Precise DGPS Applications by Establishing Reference Station Networks, Proceedings of the International Technical Meeting, ION GPS-96, Kansas City.

Received: 2007-03-29,

Reviewed: 2007-04-19, by R. Chen

Accepted: 2007-04-30. 